\title{
Aortoesophageal Fistula after Thoracic Endovascular Aortic Repair
}

Azabou N, Chaouch N, Saaidi A, Romdhane NB and Aouini F*

Department of Vascular Surgery, Military Hospital of Tunis, Tunisia

\begin{abstract}
Thoracic endovascular aortic repair (TEVAR) has become the most appropriate thoracic aortic aneurysms treatment. This approach has decreased immediate mortality and morbidity, but several progressive complications remain unknown. Herein, we report a case of a secondary aortoesophageal fistula in a 58 years old man, which came out one month after TEVAR for aneurysm.
\end{abstract}

Keywords: Thoracic endovascular aortic repair; Aortoesophageal fistula; Aneurysms; Morbidity

\section{Background}

Thoracic endovascular aortic repair (TEVAR) is increasingly applied for treatment of aortic aneurysms. Although the risk of acute complications seems to be moderate compared to open surgery, many details about mid-term and long-term morbidity and mortality are still unknown. Aortoesophageal fistula (AEF) is a rarely reported but lethal late complication after TEVAR. Physicians should be aware of this diagnosis to allow an early treatment and prevent fatal evolution.

\section{Case Report}

A 58 years old smoking man with hypertension and unspecified respiratory disease, presented to emergency department with chest pain and dyspnea. The diagnosis of myocardial infarction was first excluded. Chest X-ray was then performed showing a mediastinal widening. CT angiography (CTA) of the chest revealed a $13 \mathrm{~cm}$ saccular aneurysm of the aortic isthmus, compressing the left subclavian artery (SCA), without evidence of rupture (Figure 1). Considering the size of the aneurysm which may make open surgery more difficult, we opted for TEVAR. The procedure was performed under general anesthesia. After sizing of the stent-graft needed, a $34-150 \mathrm{~mm}$ VALIANT $^{\oplus}$ Thoracic Stent Graft with CAPTIVIA ${ }^{\bullet}$ Delivery System was used. The immediate result was satisfactory with an excluded aneurysm and no endoleaks on control angiography (Figure 2). The patient was maintained in intensive care unit for two weeks because of stroke. He also presented a fever and a decrease in the hemoglobin rate. The gastric endoscopy was normal. The CTA performed 7 days post-operatively did not show any endoleak. The patient was discharged one month after surgery with no signs of chronic bleeding or infection. He presented few hours after discharge with important hematemesis and shock. CTA revealed erosion of the esophagus by the aneurysmal sac with air bubbles into the thrombus (Figure 3). The patient was transferred in emergency to

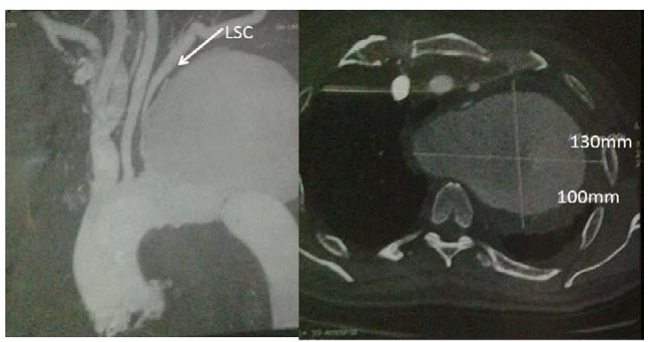

Figure 1: Aneurysm of the aortic isthmus compressing the left subclavian (LSC) artery. the operating room for surgical repair but he unfortunately died before surgery.

\section{Discussion}

AEF is a rare complication after TEVAR. The first case was reported in 1998 by Norgren [1]. Then, few cases were published; the largest series was that of Eggebrecht [2] with 6 cases. Er-Ping in his literature review, studied mechanisms of AEF complicating TEVAR [3]. To understand how this complication may occur, several hypotheses had been made:

Coverage of aortic side branches that feed the esophagus by the stent-graft lead to ischemic esophageal necrosis [2].

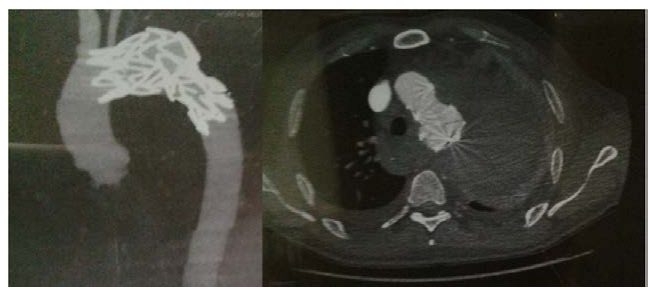

Figure 2: Excluded Aneurysm and absence of endo leaks.

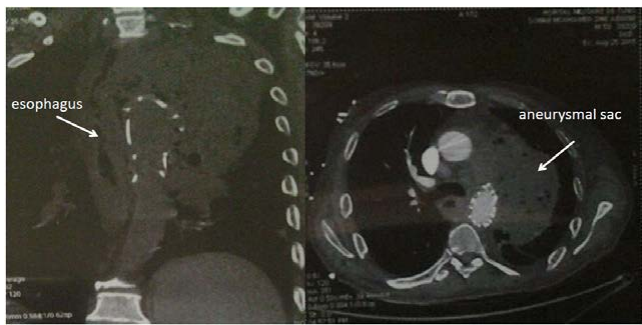

Figure 3: Erosion of the esophagus by the aneurysmal sac.

${ }^{*}$ Corresponding author: Aouini F, Department of vascular surgery, Military Hospital of Tunis; Tunisia, Tel: 71-391-133; E-mail: aouinifatma@hotmail.fr

Received July 10, 2016; Accepted September 14, 2016; Published September 25, 2016

Citation: Azabou N, Chaouch N, Saaidi A, Romdhane NB, Aouini F (2016) Aortoesophageal Fistula after Thoracic Endovascular Aortic Repair. J Vasc Med Surg 4: 287. doi: 10.4172/2329-6925.1000287

Copyright: $\odot 2016$ Azabou N, et al. This is an open-access article distributed under the terms of the Creative Commons Attribution License, which permits unrestricted use, distribution, and reproduction in any medium, provided the original author and source are credited. 
The over-sizing of the device by $10 \%$ to $20 \%$ make the aortic diameter bigger and may lead to oesophageal compression and a decrease in its lumen. The passage of the bolus in the compressed oesophagus may add injuries to this organ [4]. A mycotic aortic aneurysms [5]. An endoleak into the residual aneurysm with subsequent esophageal pressure [6]. In our case we retained as mechanism of fistula formation, the direct erosion of the esophagus by a large aneurism. Unfortunately, no necropsy was performed for an exact diagnosis of the death cause. Different therapies of AEF were reported. Some investigators reported conservative treatment with self-expanding esophageal stent placement [2] while others preferred surgical aortic repair and esophageal fistula resection via a left posterolateral thoracotomy [7]. Although its high mortality (78\%), open surgery seems to be safer [7].

\section{Conclusion}

$\mathrm{AEF}$ is an uncommon but fatal complication after TEVAR. In our reported case, it was diagnosed 30 days post-operatively but the fistula developed probably earlier. Physicians should be aware of the first symptoms such as fever and hemoglobin rate decrease to make an early diagnosis and immediate treatment.

\section{Ethic Consideration}

The consent of the family for reporting the case was obtained.

\section{References}

1. Norgren L, Jernby B, Engellau L (1998) Aortoenteric fistula caused by a ruptured stent-graft: a case report. J Endovasc Surg 5: 269-272.

2. Eggebrecht H, Mehta RH, Dechene A, Tsagakis K, Kühl H, et al. (2009) Aortoesophageal fistula after thoracic aortic stent-graft placement: a rare but catastrophic complication of a novel emerging technique. JACC Cardiovasc Interv 2: 570-576.

3. Ping Xi, Zhu J, Zhu S, Zhang Y (2014) Secondary aortoesophageal fistula after thoracic aortic aneurysm endovascular repair: literature review and new insights regarding the hypothesized mechanisms. Int J Clin Exp Med 7: 32443252.

4. Tehrani HY, Peterson BG, Katariya K, Morasch MD, Stevens R, et al. (2006) Endovascular repair of thoracic aortic tears. Ann Thorac Surg 82: 873-877.

5. Gavens E, Zaidi Z, Al-Jundi W, Kumar P (2011) Aortoesophageal fistula after endovascular aortic aneurysm repair of a mycotic thoracic aneurysm. Int J Vasc Med 649592.

6. Albors J, Bahamonde JÁ, Sanchis JM, Boix R, Palmero J (2011) Aortoesophageal fistula after thoracic stent grafting. Asian Cardiovasc Thorac Ann 19: 352-356.

7. Chiesa R, Melissano G, Marone EM, Marrocco-Trischitta MM, Kahlberg A (2010) Aorto-oesophageal and aortobronchial fistulae following thoracic endovascular aortic repair: a national survey. Eur J Vasc Endovasc Surg 39: 273-279. 